\title{
Association of the DNASE1L3 rs35677470 polymorphism with systemic lupus erythematosus, rheumatoid arthritis and systemic sclerosis: Structural biological insights
}

\author{
MARIA I. ZERVOU ${ }^{1 *}$, ATHENA ANDREOU ${ }^{2 *}$, MICHAIL MATALLIOTAKIS $^{1,3}$, \\ DEMETRIOS A. SPANDIDOS ${ }^{4}$, GEORGE N. GOULIELMOS ${ }^{1}$ and ELIAS E. ELIOPOULOS ${ }^{2}$
}

\author{
${ }^{1}$ Section of Molecular Pathology and Human Genetics, Department of Internal Medicine, School of Medicine, \\ University of Crete, 71003 Heraklion; ${ }^{2}$ Laboratory of Genetics, Department of Biotechnology, \\ Agricultural University of Athens, 11855 Athens; ${ }^{3}$ Third Department of Obstetrics and Gynaecology, \\ Aristotle University of Thessaloniki, 54124 Thessaloniki; ${ }^{4}$ Laboratory of Clinical Virology, \\ School of Medicine, University of Crete, 71003 Heraklion, Greece
}

Received March 28, 2020; Accepted June 30, 2020

DOI: $10.3892 / \mathrm{mmr} .2020 .11547$

\begin{abstract}
Although genome-wide association studies (GWAS) have identified hundreds of autoimmune disease-associated loci, much of the genetics underlying these diseases remains unknown. In an attempt to identify potential causal variants, previous studies have determined that the rs 35677470 missense variant of the Deoxyribonuclease I-like 3 (DNASEIL3) gene was associated with the development of systemic lupus erythematosus (SLE), rheumatoid arthritis (RA) and systemic sclerosis (SSc). DNase1L3 is a member of the human DNase I family, representing a nuclease that cleaves double-stranded DNA during apoptosis and serving a role in the development of autoimmune diseases. The present study aimed to determine the role of the rs 35677470 variant at the DNASE1L3 gene leading to the R206C mutation in SLE, RA and SSc. The underlying mechanism potentially affecting protein structure loss of function was also assessed. DNASEIL3 evolution was investigated to define conservation elements in the protein sequence. Additionally, 3D homology modeling and in silico mutagenesis was performed to localize the polymorphism under investigation. Evolutionary analysis revealed heavily conserved sequence elements among species, indicating structural/functional importance. In silico mutagenesis and
\end{abstract}

Correspondence to: Dr Maria I. Zervou, Section of Molecular Pathology and Human Genetics, Department of Internal Medicine, School of Medicine, University of Crete, University Campus of Voutes, 71003 Heraklion, Greece

E-mail:mzervou@med.uoc.gr

${ }^{*}$ Contributed equally

Key words: autoimmune diseases, gene polymorphism, three-dimensional model, in silico mutagenesis, deoxyribonuclease I-like 3 , single nucleotide polymorphism
3D protein structural analysis also demonstrated the potentially varied impact of the DNASEIL3 (rs35677470) single nucleotide polymorphism (SNP), providing an explanation for its effect on the R206C variant. Structural analysis demonstrated that the rs35677470 SNP encodes a non-conservative amino acid variation, $\mathrm{R} 206 \mathrm{C}$, which disrupted the conserved electrostatic network holding secondary protein structure elements in position. Specifically, the R206 to E170 interaction forming part of a salt bridge network stabilizing two $\alpha$-helices was interrupted, thereby affecting the molecular architecture. Previous studies on the effect of this SNP in Caucasian populations demonstrated lower DNAse1L3 activity levels, which is consistent with the current results. The present study comprehensively evaluated the shared autoimmune locus of DNASEIL3 (rs35677470), which produced an inactive form of DNaseIL3. Furthermore, structural analysis explained the potential role of the produced mutation by modifying the placement of structural elements and consequently introducing disorder in protein folding, affecting biological function.

\section{Introduction}

The genes and mechanisms involved in complex autoimmune diseases, which affect $\sim 5 \%$ of the population, remain obscure. Genome-wide association studies (GWAS) have identified hundreds of autoimmune disease-associated loci without defining causal variants (1). Understanding the mechanisms that underlie these diseases may contribute to the development of diseases-modifying therapeutic protocols. Accumulating evidence has revealed that common genetic factors may predispose individuals to multiple autoimmune diseases. In this framework, several genetic polymorphisms have been associated with systemic lupus erythematosus (SLE) and other autoimmune diseases (2). In addition, it has been reported that most of the identified systemic scleroderma (SSc) susceptibility loci overlap with those of other autoimmune diseases and in particular, disorders such as SLE and rheumatoid arthritis (RA) (3). Thus, previous studies have supported the 
hypothesis that common deregulated pathways exist across multiple autoimmune diseases.

In an attempt to identify potential causal variants that alter physiologic pathways and are involved in susceptibility to autoimmune diseases, various studies have revealed that the missense variant rs 35677470 at the deoxyribonuclease I-like 3 (DNASE1L3) locus was associated with the development of SLE $(4,5)$, RA (6) and SSc (7), thus representing a shared risk factor for these diseases. However, the exact functional consequence of this polymorphism has not yet been thoroughly investigated. Deoxyribonuclease I-like 3 (DNase 1L3) is a member of the human DNase I family, representing a nuclease that cleaves double-stranded DNA during apoptosis (8). The protein encoded by DNASE1L3 is one of three human homologs of DNase I and functions as an endonuclease capable of cleaving both single- and double-stranded DNA (9-11).

SLE is a chronic, severe, multiorgan systemic autoimmune disease that predominantly affects women, with a complex genetic inheritance and strong clustering in families (4). It is characterized by the production of high titers of autoantibodies directed against native DNA, cell surface and other cellular constituents that are associated with high morbidity rates (12). RA is a chronic, systemic multifactorial disease of unknown etiology, characterized by progressive joint destruction resulting in severe disability. It results from a complex interplay between genetic and environmental factors $(13,14)$. $\mathrm{SSc}$ is a chronic, multisystem autoimmune disease clinically characterized by progressive skin and internal organ fibrosis, exhibiting one of the highest mortality rates among rheumatic diseases, leading to premature death in affected individuals $(15,16)$. SSc affects connective tissue and produces various heterogeneous clinical manifestations, including inflammation, autoimmunity, vasculopathy and excessive extracellular matrix production and deposition (17). SSc, RA and SLE are heterogeneous diseases of the connective tissue that share clinical and epidemiological manifestations as well as life-threatening complications (18). Considering that the production of autoantibodies represents a main feature of these diseases, patients often exhibit an extensive deregulation of the innate and adaptive immune response (19).

Given that autoimmune diseases may share common susceptibility genes, potentially leading to the development of shared therapeutic approaches, the present study aimed to investigate the potential role of the rs 35677470 variant at the DNASE1L3 gene and the resultant R206C mutation in the development of SLE, RA and SSc. The underlying mechanism potentially affecting protein structure loss of function was also assessed.

\section{Materials and methods}

Sequence retrieval. Protein sequences were retrieved from the UNIPROT database (20) and the structural information was obtained from the Protein Data Bank (21). To find homologs across species, BLAST searches were performed with Mega BLAST (National Center for Biotechnology Information) using the protein databases PDB and SwissProt and Blastp (protein-protein BLAST) with default parameters (22).

Sequence alignment. T-COFFEE, the multiple sequence alignment program (23), was used to perform all protein sequence alignments. Default parameters were used for the alignment. ESPript was used to depict the sequence alignments and to incorporate the available conservation or diversity information and ENDscript was used for 3D homology representation $(24,25)$.

Phylogenetic analysis. To test for phylogeny, trees were constructed using maximum-likelihood analysis with 500 bootstrap replications (26) implemented by MEGA7 (27). The evolutionary tree was inferred from protein sequences using the Maximum Likelihood method (28), based on the Jones-Taylor-Thorntonmatrix model (28) and incorporated using the Gamma distribution model. The tree with the highest log likelihood was presented in all phylogeny figures. Initial trees for heuristic searches were obtained automatically by applying Neighbor-Join (29) and BioNJ (30) algorithms to a matrix of pairwise distances, estimated using the Maximum Composite Likelihood (31) approach and then by selecting the topology with the superior log likelihood value.

Structural analysis. The structure of the DNASE1L3 protein (Homo_sapiens_NP_004935.1) was built using homology modeling with the SWISSMODEL program via the EXPASY server (32) and was based on the experimentally determined crystal structures of homologous proteins retrieved from the Protein Data Bank (IDs 1atn, 4awn and 3w3d) (33-35). In silico mutagenesis of the Arg206Cys point mutation was created in the homology model of the wild-type DNASE1L3 protein structure using the PyMOL molecular-graphics system V.2.2 (Schrödinger, LLC) (36). Multiple structure alignments were performed using PyMOL (36) based on protein backbone RMSD (Root Mean Square Deviation) optimization and sequence alignment. ENDscript (25) was used to depict weak and strong sequence conservations in structures across species in order to pinpoint variable sites on the functional domain. All figures depicting 3D models were generated using the PyMOL molecular-graphics system V.2.2 (Schrödinger, LLC) (36).

\section{Results and Discussion}

Phylogenetics and sequence analysis. DNASE1L3 evolution was investigated to define conservation elements in its protein sequence. Evolutionary analysis is used to identify positions on the protein sequences that are heavily conserved across species, indicating structural importance (37). To determine the evolutionary relationship among DNase1L3 members of the DNase I family and to identify additional homologue DNAasesI in other species, exhaustive BLAST searches were performed using a variety of available protein sequence databases. Using multiple phylogenetic approaches, the results revealed high sequence conservation among species, with separation only occurring across different animal taxa. Outgroups (mollusks and bacteria) were aligned with high confidence, demonstrating good preservation of important secondary structure elements (Figs. 1 and 2).

Structural analysis. The current study utilized 3D homology modeling to localize polymorphisms in the DNASE1L3 protein. Structural analysis revealed that the rs35677470 DNASE1L3 single nucleotide polymorphism (SNP), 


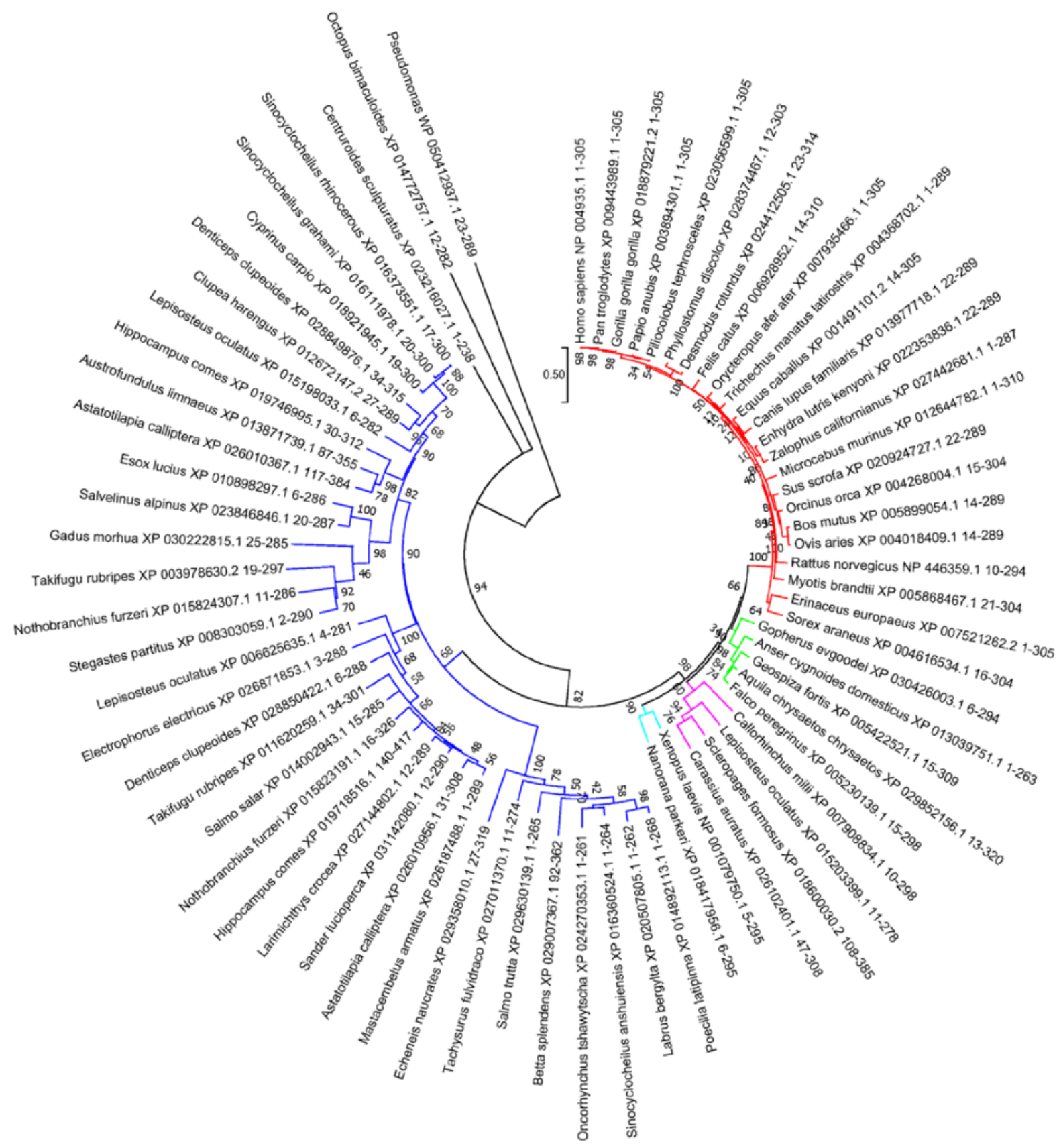

Figure 1. Molecular phylogenetic analysis of DNAseI across species. The unrooted tree of 72 protein sequences selected using the Maximum Likelihood method is presented. The sequences are clustered according to animal groups represented by different colors (Mammals in red, birds in green, freshwater fish in purple, reptiles in cyan and fish in blue). Outgroups (octopus and bacteria) are in black.

which encodes the non-conservative amino acid variation Arg206Cys, modified the conserved electrostatic network that holds protein secondary structure elements in position (Fig. 3A). Specifically, the guanidinium group of the Arg206 side chain interacted with the carboxylate group of Glu170, forming a strong salt bridge (Fig. 3B). Together with the Arg208 to Asp219 charge interaction, an electrostatic salt bridge network was formed, which stabilized two important scaffold $\alpha$-helices. This network was interrupted by the highly defective rs35677470 SNP allele, in which arginine is replaced by a cysteine, affecting the molecular architecture (Fig. 3C and Table I). Previous studies on the effect of this SNP in Caucasian populations, resulting in a lower level of DNAse1L3 activity, are consistent with this observation, demonstrating that the SNP affects position 206 in the protein, thus producing a less active form of human DNAse1L3 $(38,39)$. The introduction of the cysteine residue at position 206 has no effect on disulphide bond formation, since all four cystines forming disulphide bridges (24-52 and 194-231; Fig. 3) are distal to position 206 and sterically inaccessible at this position. Herein, the structural analysis showed that although position 206 is distant to the nucleotide binding site forming residues, the effect of this mutation destabilizes the nucleotide interacting loop preceding that of position 206 (residue 193-196). The enhanced mobility of this loop facing the nucleotide recognition site and the subsequent direct interaction with the affected attacked nucleotide may affect the enzymatic activity of DNAselL3. 

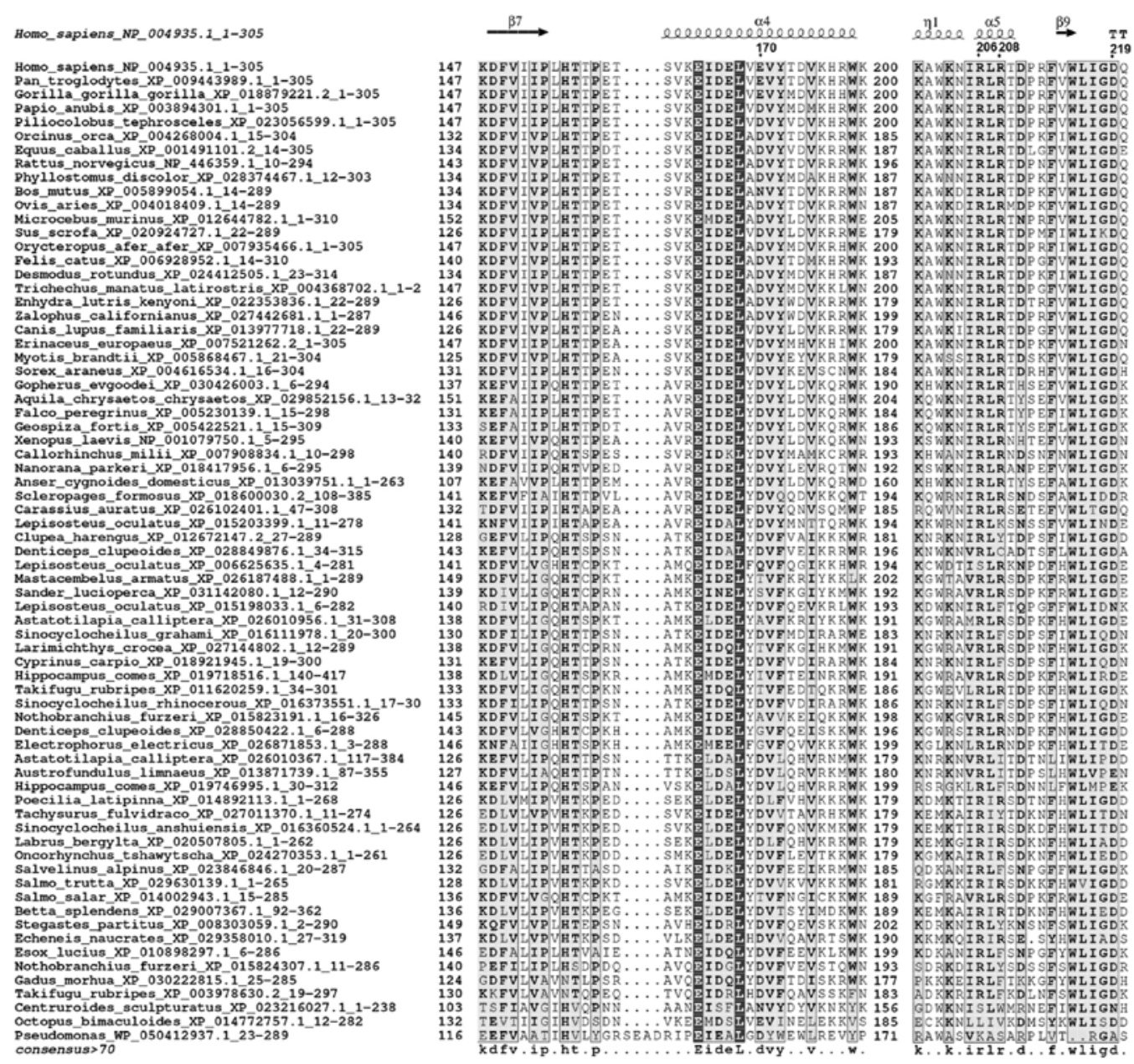

Figure 2. Partial representation of cross species sequence alignment for elements of the secondary structure containing the position of the stabilizing salt bridge network counterparts E170 to R206 and R208 to D219 (indicated by black dots) in human DNAseIL3. Gray-scale shading indicates amino acid residue conservation.

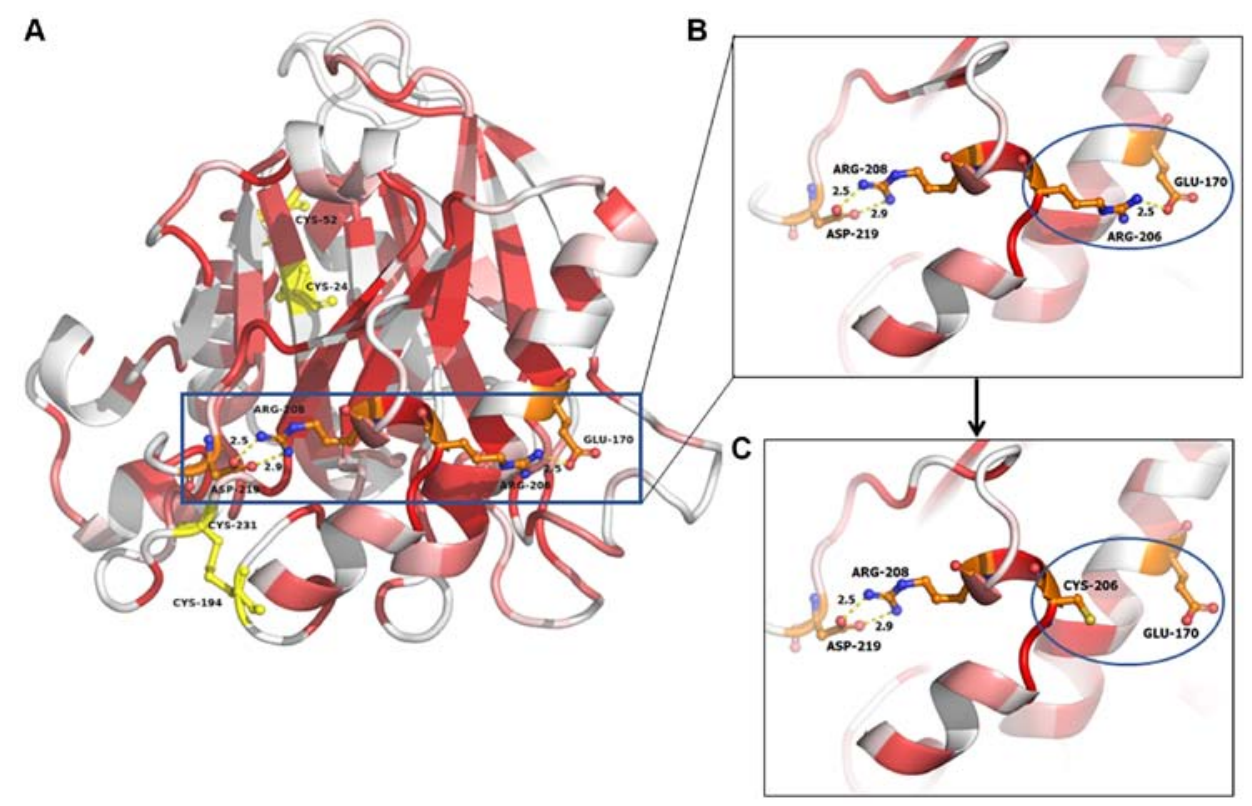

Figure 3. Ribbon representation of the DNAase1L3 homology model. Sequence conservation (red indicating most conserved and white representing least conserved) among the sequences studied, the position of the stabilizing salt bridge network (Glu170 to Arg206 and Arg208 to Asp219; framed by blue rectangle) and the disulphide bridges (depicted with Cys residues colored in yellow are shown). (A) Close-up view of the residues of the native structure involved in the salt bridge network. The residues are shown in stick representation (colored in orange) and interactions are shown as yellow dashed lines. (B) The location of Arg206 and Glu170 interaction is highlighted by the blue oval. The disrupted salt bridge network with the Arg206Cys mutation are presented. (C) The disrupted salt bridge network by Arg206Cys mutation is presented in the blue oval. Distances (yellow dashed lines) are presented in Angstroms. 
Table I. Summary of salt bridge stabilizing interactions.

\begin{tabular}{lcc}
\hline Salt bridge interaction & Native & $\begin{array}{r}\text { rs35677470 DNASE1L3 } \\
\text { SNP(Arg206Cys) }\end{array}$ \\
\hline Arg206 $\rightarrow$ Glu170 & + & - \\
Arg208 $\rightarrow$ Asp219 & + & + \\
\hline
\end{tabular}

DNASE1L3, deoxyribonuclease I-like 3 gene; SNP, single nucleotide polymorphism.

Table II. Role overview of the rs35677470 (Arg206Cys) single nucleotide polymorphism in autoimmune diseases.

\begin{tabular}{|c|c|c|}
\hline Disease & Effect & Author (Refs.) \\
\hline SLE & Risk & $\begin{array}{l}\text { Harley et al 2008, (4) } \\
\text { Gateva et al 2009, (5) }\end{array}$ \\
\hline RA & Risk & Westra et al 2018, (6) \\
\hline $\mathrm{SSc}$ & Risk & $\begin{array}{l}\text { Martin et al 2012, (3) } \\
\text { Zochling et al 2014, (7) } \\
\text { Mayes et al 2014, (41) }\end{array}$ \\
\hline T1D & No genetic association & Westra et al 2018,(6) \\
\hline
\end{tabular}

SLE, systemic lupus erythematosus; RA, rheumatoid arthritis; $\mathrm{SSc}$, systemic sclerosis, T1D, type 1 diabetes.

Arg206Cys mutation of DNASE1L3 in patients with SLE, RA and SSc. The potentially causal missense variant rs35677470 localized to the DNASE1L3 gene (exon 8) at position 3p14.3 was identified following GWAS in patients with SLE, RA and SSc (Table II). However, despite exhibiting a pleiotropic effect in autoimmunity, this SNP was not found to be associated with Type 1 Diabetes (6). A second SNP (rs7652027) of the DNASEIL3 genomic region has also been recently associated with SSc (40). However, the same study revealed no association between the rs35677470 SNP with SSc due to the absence of the SNP from the panel used. In other studies, by Mayes et al (41) and Zochling et al (7), the association signal of the rs35677470 SNP was also significantly associated with anti-centromere-antibody-positive $\left(\mathrm{ACA}^{+}\right)$patients with SSc but not with ACA individuals. Therefore, it has been hypothesized that the association between ACA and SSc in the case of rs35677470 loss-of-function DNASEIL3 variants may provide a link between defective apoptotic DNA breakdown and ACA production (41).

In regards to the potential role of the rs35677470 SNP in SLE and taking into account that SLE pathogenesis is associated with a reduced ability to clear DNA released from apoptotic cells, Al-Mayouf et al (42) hypothesized that DNASEIL3 gene dysfunction may lead to impaired DNA breakdown and clearance from apoptotic cells, thus resulting in the formation of antibodies recognizing DNA and immune complexes. Furthermore, given that similar types of DNA-driven immune complexes (such as anti-nuclear and ACA antibodies) are also characteristic of SSc, this hypothesis is also applicable in SLE (7).
Recently, decreased expression levels of DNASE113 have been detected in patients with SLE, resulting in the reduction of nucleosome DNA digestion in serum, which is associated with the development of anti-double stranded DNA antibodies. However, the expression and activity of DNASE113 remained unchanged in patients with RA, strengthening the hypothesis that the immunopathological mechanism of RA differs from that of SLE (43). Furthermore, Serpas et al (44) targeted the DNASE1L3 enzyme as a potential therapeutic agent for SLE. To this end, a murine model with DNASE1L3 deficiency was developed, in which mice exhibit features of SLE and renal disease. Methods to treat this disease by targeting the DNASE1L3 enzyme are currently being investigated.

Candidate gene studies and GWAS have attempted to partially elucidate the complex genetic architecture of SLE through the identification of $>90$ risk loci (45) and to determine the existing differences in risk variants across different continents (46). These studies have established the importance of several pathways in SLE, including innate immune responses, the activation of lymphocytes and immune complex clearing $(45,47-49)$. The present study comprehensively evaluated rs 35677470 of the shared autoimmune locus DNASE1L3, which has been reported to produce an inactive form of DNaseIL3 $(38,39)$. Structural analysis performed in the current study elucidated the potential role of mutations that modify the placement of structural elements, consequently producing disordered protein folding and thereby affecting its biological function. In the post-genomic era, the identification of genes and molecules involved in the molecular mechanisms of various diseases is of vital importance to elucidate clinically relevant functional defects, thus contributing to drug discovery and the production of novel therapies. Therefore, determining the 3D structure and analyzing the role of any causative mutation in the pathogenesis of disease, from the structural/functional point of view, is necessary. As a consequence, potential abnormalities in the conformation and activity of proteins can be elucidated. Additionally, an efficient integration of these data with other biological factors such as antibodies, antagonists, inhibitors and binders (50) may identify novel targets for pharmaceutical intervention. The results of the present study further elucidated the biological significance of rs35677470 at the DNASE1L3 locus in SLE, RA and SSc, and demonstrated the value of pleiotropic gene studies for autoimmune diseases.

\section{Acknowledgements}

Not applicable.

\section{Funding}

This study was supported by the project 'INSPIRED: The National Research Infrastructures on Integrated Structural Biology, Drug Screening Efforts and Drug Target Functional Characterization' (grant no. MIS 5002550), which is implemented under the Action 'Reinforcement of the Research and Innovation Infrastructure', funded by the Operational Program 'Competitiveness, Entrepreneurship and Innovation' (NSRF 2014-2020) and co-financed by Greece and the European Union (European Regional Development Fund). 


\section{Availability of data and materials}

The datasets used and/or analyzed during the present study are available from the corresponding author on reasonable request.

\section{Authors' contributions}

EEE, MIZ and GNG designed the current study and drafted the manuscript. AA, GNG, MM, DAS and MIZ searched the literature. EEE, MM, DAS, AA and MIZ analyzed and interpreted the data. MIZ, AA and DAS critically revised the manuscript. All authors have read and approved the final manuscript.

\section{Ethics approval and consent to participate}

Not applicable.

\section{Patient consent for publication}

Not applicable.

\section{Competing interests}

DAS is the Managing Editor of the journal, but had no personal involvement in the reviewing process, or any influence in terms of adjudicating on the final decision, for this article. All other authors declare that they have no competing interests.

\section{References}

1. Goulielmos GN, Zervou MI, Myrthianou E, Burska A Niewold TB and Ponchel F: Genetic data: The new challenge of personalized medicine, insights for rheumatoid arthritis patients. Gene 583: 90-101, 2016

2. Ramos PS, Criswell LA, Moser KL, Comeau ME, Williams AH, Pajewski NM, Chung SA, Graham RR, Zidovetzki R, Kelly JA, et al: A comprehensive analysis of shared loci between systemic lupus erythematosus (SLE) and sixteen autoimmune diseases reveals limited genetic overlap. PLoS Genet 7: e1002406, 2011

3. Martín JE, Bossini-Castillo L and Martín J: Unraveling the genetic component of systemic sclerosis. Hum Genet 131: $1023-1037,2012$

4. International Consortium for Systemic Lupus Erythematosus Genetics (SLEGEN); Harley JB, Alarcón-Riquelme ME, Criswell LA, Jacob CO, Kimberly RP, Moser KL, Tsao BP, Vyse TJ, Langefeld CD, et al: Genomewide association scan in women with systemic lupus erythematosus identifies susceptibility variants in ITGAM, PXK, KIAA1542 and other loci. Nat Genet 40: 204-210, 2008.

5. Gateva V, Sandling JK, Hom G, Taylor KE, Chung SA, Sun X, Ortmann W, Kosoy R, Ferreira RC, Nordmark G, et al: A large-scale replication study identifies TNIP1, PRDM1, JAZF1, UHRF1BP1 and IL10 as risk loci for systemic lupus erythematosus. Nat Genet 41: 1228-1233, 2009.

6. Westra HJ, Martínez-Bonet M, Onengut-Gumuscu S, Lee A, Luo Y, Teslovich N, Worthington J, Martin J, Huizinga T, Klareskog L, et al: Fine-mapping and functional studies highlight potential causal variants for rheumatoid arthritis and type 1 diabetes. Nat Genet 50: 1366-1374, 2018.

7. Zochling J, Newell F, Charlesworth JC, Leo P, Stankovich J, Cortes A, Zhou Y, Stevens W, Sahhar J, Roddy J, et al: An ImmunoChip-based interrogation of scleroderma susceptibility variants identifies a novel association at DNASE1L3. Arthritis Res Ther 16: 438, 2014.

8. Sisirak VB, Sally B, D'Agati V, Martinez-Ortiz W, Özçakar ZB, David J, Rashidfarrokhi A, Yeste A, Panea C, Chida AS, et al: Digestion of chromatin in apoptotic cell microparticles prevents autoimmunity. Cell 166: 88-101, 2016.
9. Rodriguez AM, Rodin D, Nomura H, Morton CC, Weremowicz S and Schneider MC: Identification, localization, and expression of two novel human genes similar to deoxyribonuclease I. Genomics 42: 507-513, 1997.

10. Zeng Z, Parmelee D, Hyaw H, Coleman TA, Su K, Zhang J, Gentz R, Ruben S, Rosen C and Li Y: Cloning and characterization of a novel human DNase. Biochem Biophys Res Commun 23: 499-504, 1997.

11. Chen WJ, Lee IS, Chen CY and Liao TH: Biological functions of the disulfides in bovine pancreatic deoxyribonuclease. Protein Sci 13: 875-883, 2004

12. Helmick CG, Felson DT, Lawrence RC, Gabriel S, Hirsch R, Kwoh CK, Liang MH, Kremers HM, Mayes MD, Merkel PA, et al: Estimates of the prevalence of arthritis and other rheumatic conditions in the United States. Part I. Arthritis Rheum 58: 15-25, 2008.

13. Firestein GS: Evolving concepts of rheumatoid arthritis. Nature 423: 356-361, 2003.

14. McAllister KM, Eyre S and Orozco G: Genetics of rheumatoid arthritis: GWAS and beyond. Open Access Rheumatology Res Rev 3: 31-46, 2013.

15. Gabrielli A, Avvedimento EV and Krieg T: Scleroderma. N Engl J Med 360: 1989-2003, 2009.

16. Barnes J and Mayes MD: Epidemiology of systemic sclerosis: Incidence, prevalence, survival, risk factors, malignancy, and environmental triggers. Curr Opin Rheumatol 24: 165-170, 2012.

17. Chizzolini C: T cells, B cells, and polarized immune response in the pathogenesisof fibrosis and systemic sclerosis. Curr Opin Rheumatol 20: 707-712, 2008.

18. Wallace B, Vummidi D and Khanna D: Management of connective tissue diseases associated interstitial lung disease: A review of the published literature. Curr Opin Rheumatol 28:236-245, 2016.

19. Liu $\mathrm{Z}$ and Davidson A: Taming lupus-a new understanding of pathogenesis is leading to clinical advances. Nat Med 18: 871-882, 2012.

20. UniProt Consortium: UniProt: A worldwide hub of protein knowledge, Nucleic Acids Res 47: D506-D515, 2019.

21. Berman HM, Westbrook J, Feng Z, Gilliland G, Bhat TN, Weissig H, Shindyalov IN and Bourne PE: The protein data bank. Nucleic Acids Res 28: 235-242, 2000.

22. Altschul SF, Madden TL, Schäffer AA, Zhang J, Zhang Z, Miller W and Lipman DJ: Gapped BLAST and PSI-BLAST: A new generation of protein database search programs. Nucleic Acids Res 25: 3389-3402, 1997.

23. Notredame C, Higgins DG and Heringa J: A novel method for fast and accurate multiple sequence alignment. Mol Biol 302: 205-217, 2000.

24. Robert X and Gouet P: Deciphering key features in protein structures with the new ENDscript server. Nucleic Acids Res 42 (Web Server Issue): W320-W324, 2014.

25. Gouet P, Robert X and Courcelle E: ESPript/ENDscript: Extracting and rendering sequence and 3D information from atomic structures of proteins. Nucleic Acids Res 31: 3320-3323, 2003.

26. Felsenstein J: Confidence limits on phylogenies: An approach using the bootstrap, Evolution 39: 783-791, 1985.

27. Kumar S, Stecher G and Tamur K: MEGA7: Molecular evolutionary genetics analysis version 7.0 for bigger datasets. Mol Biol Evol 33: 870-1874, 2016.

28. Felsenstein J: Evolutionary trees from DNA sequences: A maximum likelihood approach. J Mol Evol 17: 368-376, 1981.

29. Saitou N and Nei M: The neighbor-joining method: A new method for reconstructing phylogenetic trees. Mol Biol Evol 4: 406-425, 1987.

30. Gascuel O: BIONJ: An improved version of the NJ algorithm based on a simple model of sequence data. Mol Biol Evol 14: 685-695, 1997.

31. Tamura K, Nei M and Kumar S: Prospects for inferring very large phylogenies by using the neighbor-joining method. Proc Natl Acad Sci USA 101:11030-11035, 2004.

32. Waterhouse A, Bertoni M, Bienert S, Studer G, Tauriello G, Gumienny R, Heer FT, de Beer TAP, Rempfer C, Bordoli L, et al: SWISS-MODEL: Homology modelling of protein structures and complexes. Nucleic Acids Res 46: W296-W303, 2018.

33. Kabsch W, Mannherz HG, Suck D, Pai EF and Holmes KC: Atomic structure of the actin: DNase I complex. Nature 347: 37-44, 1990. 
34. Parsiegla G, Noguere C, Santell L, Lazarus RA and Bourne Y: The structure of human DNase I bound to magnesium and phosphate ions points to a catalytic mechanism common to members of the DNase I-like superfamily. Biochemistry 51: 10250-10258, 2012.

35. Sasaki K, Sakabe K, Sakabe N, Kondo H and Shimomura M: Refined structure and solvent network of chicken gizzard G-actin DNase 1 complex at $1.8 \AA$ resolution. Acta Cryst A49: C111-C112, 1993.

36. The PyMOL molecular graphics system, version 1.7.4, Schrödinger, LLC, Available online, http://www.pymol.org.

37. Andreou A, Giastas P, Christoforides E and Eliopoulos EE: Structural and evolutionary insights within the polysaccharide deacetylase gene family of Bacillus anthracis and Bacillus cereus. Genes (Basel) 9: 386, 2018.

38. Ueki M, Takeshita H, Fujihara J, Iida R, Yuasa I, Kato H, Panduro A, Nakajima T, Kominato $Y$ and Yasuda $T$ : Caucasian-specific allele in non-synonymous single nucleotide polymorphisms of the gene encoding deoxyribonuclease I-like 3 , potentially relevant to autoimmunity, produces an inactive enzyme. Clin Chim Acta 407: 20-24, 2009.

39. Ueki M, Fujihara J, Takeshita H, Kimura-Kataoka K, Iida R, Yuasa I, Kato H and Yasuda T: Global genetic analysis of all single nucleotide polymorphisms in exons of the human deoxyribonuclease I-like 3 gene and their effect on its catalytic activity. Electrophoresis 32: 1465-1472, 2011.

40. López-Isac E, Acosta-Herrera M, Kerick M, Assassi S, Satpathy AT, Granja J, Mumbach MR, Beretta L, Simeón CP, Carreira P, et al: GWAS for systemic sclerosis identifies multiple risk loci and highlights fibrotic and vasculopathy pathways. Nat Commun 10: 4955, 2019.

41. Mayes MD, Bossini-Castillo L, Gorlova O, Martin JE, Zhou X, Chen WV, Assassi S, Ying J, Tan FK, Arnett FC, et al: Immunochip analysis identifies multiple susceptibility loci for systemic sclerosis. Am J Hum Genet 94: 47-61, 2014.

42. Al-Mayouf SM, Sunker A, Abdwani R, Abrawi SA, Almurshedi F, Alhashmi N, Al Sonbul A, Sewairi W, Qari A, Abdallah E, et al: Loss-of-function variant in DNASE1L3 causes a familial form of systemic lupus erythematosus. Nat Genet 43: 1186-1188, 2011.
43. Zhao Q, Yang C, Wang J, Li Y and Yang P: Serum level of DNase113 in patients with dermatomyositis/polymyositis, systemic lupus erythematosus and rheumatoid arthritis, and its association with disease activity. Clin Exp Med 17: 459-465, 2017.

44. Serpas L, Chan RWY, Jiang P, Ni M, Sun K, Rashidfarrokhi A, Soni C, Sisirak V, Lee WS, Cheng SH, et al: Dnase113 deletion causes aberrations in length and end-motif frequencies in plasma DNA. Proc Natl Acad Sci USA 11: 641-649, 2019.

45. Moser K, Kelly J, Lessard C and Harley JB: Recent insights into the genetic basis of systemic lupus erythematosus. Genes Immun 10: 373-379, 2009.

46. Goulielmos GN, Zervou MI, Vazgiourakis VM, Ghodke-Puranik Y, Garyfallos A and Niewold TB: The genetics and molecular pathogenesis of systemic lupus erythematosus (SLE) in populations of different ancestry. Gene 668: 59-72, 2018.

47. Niewold TB: Interferon alpha-induced lupus: Proof of principle. J Clin Rheumatol 14: 131-132, 2008.

48. Ko K, Koldobskaya Y, Rosenzweig E and Niewold TB: Activation of the interferon pathway is dependent upon autoantibodies in African-American SLE patients, but not in European-American SLE patients. Front Immunol 4: 309, 2013.

49. Rahman A and Isenberg DA: Systemic lupus erythematosus. N Engl J Med 358: 929-939, 2008.

50. Márquez A, Kerick M, Zhernakova A, Gutierrez-Achury J, Chen WM, Onengut-Gumuscu S, González-Álvaro I, RodriguezRodriguez L, Rios-Fernández R, González-Gay MA, et al: Meta-analysis of Immunochip data of four autoimmune diseases reveals novel single-disease and cross-phenotype associations. Genome Med 10: 97, 2018.

(i) (9) This work is licensed under a Creative Commons Attribution-NonCommercial-NoDerivatives 4.0 International (CC BY-NC-ND 4.0) License. 